\title{
The Principle of Polysaccharide Gels
}

\author{
Masakuni Tako1,2 \\ ${ }^{1}$ Department of Subtropical Bioscience and Biotechnology, University of the Ryukyus, Nishihara, Okinawa, Japan \\ ${ }^{2}$ Present Address: Health and Longevity Research Laboratory, Integrated Innovation Research Center, \\ University of the Ryukyus, Nishihara, Okinawa, Japan \\ Email: tako@agr.u-ryukyu.ac.jp
}

Received 5 January 2015; accepted 20 January 2015; published 27 January 2015

Copyright (C) 2015 by author and Scientific Research Publishing Inc.

This work is licensed under the Creative Commons Attribution International License (CC BY). http://creativecommons.org/licenses/by/4.0/

(c) (i) Open Access

\section{Abstract}

For several decades attention has been directed to natural polysaccharide gels and synthesized polymer gels. The structure-function relationships at molecular level in water of polysaccharides, $\boldsymbol{\kappa}$-carrageenan, $\iota$-carrageenan, agarose (agar), and gellan family of polysaccharides (gellan, welan, rhamsan, S-657, deacetylated rhamsan and native gellan gum), which are industrially useful polysaccharides extracted from family of red seaweeds and bacteria, in principle are discussed on the view point of rheological aspects. The polysaccharide molecules $(0.1 \%-1.0 \%)$ play a dominant role in the center of the tetrahedral cavities occupied by water molecules $(99.0 \%-99.9 \%)$, and the arrangement is similar to a tetrahedral structure in a gelation process. The cage and hydrophobic effect play thermal dynamically dominant role in gelation process which gives lowest entropy to electrons of sugar residues. Though the chemical structure of these polysaccharides similar each other, their rheological (gelling) characteristics are quite different. Many investigations about the gelling properties of the polysaccharides have been undertaken to elucidate the structure-function relationship, but no other researchers have established mechanism at the molecular level. There is consistency in our investigations. Thus, the rheological analysis is one of significant methods for understanding the structure-function relationship of polysaccharides in aqueous media. The discussion provides many important information not only in academic field, but also in industrial one, such as food, cosmetic, pharmaceutical, drug delivery and tissue industries, and biotechnology.

\section{Keywords}

Polysaccharide Gels, Principle, Gelation Mechanism of Polysaccharides, Hydrogen Bonding, Ionic Bonding, Van der Waals Forces of Attraction

\section{Introduction}

Gels are solid, jelly-like materials formed from colloid polysaccharides, proteins and synthesized polymers. Gels 
have been applied in the food, cosmetic, paint, pharmaceutical, photographic, petroleum and chemical industries, and have additional potential uses in catalysis, separation of materials, drug delivery, and tissue engineering. Recently, many applications of polysaccharide and synthesized polymer conjugated gels are discovered because of the gel's ability to trap and immobilize functional agents, such as enzymes, pharmaceuticals, tissue engineering and other bioactive agents. Specifically, some polysaccharides are paid attentions to develop in biomedical applications [1]-[3].

Polysaccharides that are produced commercially from seaweeds, plants, animals and micro-organisms have been used in food, cosmetic, paper, textile, oil, pharmaceutical, medical, and other industries as viscous enhancing, water-holding, emulsifying, oil-drilling, coating, encapsulating, protective, gelling and other agents. Specifically, for several decades attention has been directed to polysaccharide gels. Therefore, we previously investigated the structure-function relationship [4] and gelation mechanism of polysaccharides in aqueous solutions at a molecular level using a rheogoniometer, polarimeter and ${ }^{1} \mathrm{H}-\mathrm{NMR}$ spectrometer. The polysaccharides, such as $\kappa$-carrageenan [5]-[7], $t$-carrageenan [8] [9], agarose (agar) [10], gellan gum [11] [12], amylose [13] [14], curdlan [15], alginate [16] [17], and deacetylated rhamsan gum [18], in water changed into an ice-like structure with hydrogen bonding between polymer and water molecules, and between water-water molecules even at a concentration range of $0.1 \%-1.0 \%(\mathrm{~W} / \mathrm{V})$ at room temperature, resulting in gelation. Such dramatic changes from liquid into gels have been understood at the molecular level in principle [19]. Amylopectin (rice) [20]-[22] and starch (rice, potato and wheat) gelatinization [23]-[27] and retrogradation [26] [28] processes including water molecules were also discussed at molecular level in principle [29].

We review herein the gelation mechanism of $k$-carrageenan [5]-[7], $l$-carrageenan [8] [9], agarose(agar) [10] and gellan family of polysaccharides [11] [12] [30]-[33] which are industrial useful polymers and discuss their structure-gelling relationship in principle. Though the chemical structure of these polysaccharides similar each other (Figure 1), their rheological (gelling) characteristics are quite different.

\section{Gelation Mechanism of Polysaccharides}

As metioned above, we proposed gelation mechanism for $\kappa$-carrageenan [5]-[7], $t$-carrageenan [8] [9], agarose (agar) [10], gellan gum [11] [12], amylose [13] [14], curdlan [15], alginate [16] [17], and deacetylated rhamsan gum [18] at the molecular level. Though xanthan gum which is one of bacterial polysaccharides produced commercially by Xanthomonas campestris does no gel alone, but shows curious rheological characteristics [34]-[39] and forms gel in a mixture solution with galactomannan [40]-[46] and glucomannan [47] [48]. We proposed cogelation mechanism of xanthan and galactomannans (locust-bean gum [40]-[42], guar gum [43], tara-bean gum [44], Leucaena gum [45], Leonix gum [46], and konjac glucomannan [47] [48]. A little interaction was observed in a mixture of high galactose content guar gum (33\%) or Leucaena gum (40\%). The results indicated that the
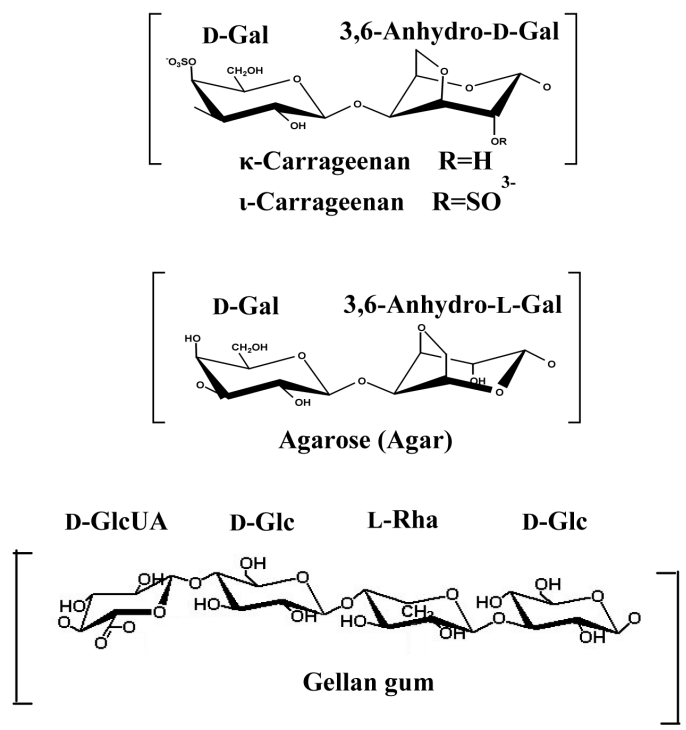

Figure 1. Chemical structure of carrageenans, agarose and gellan gum. 
weak interaction was due to the presence of side-chains of the galactomannan molecules. These side-chains prevented the insertion of the charged trisaccharide side-chains of the xanthan molecules into the galactomannan molecules. More strong co-gelation than that of galactomannan (locust-bean gum) occurred in a mixture solution of xanthan and konjac glucomannan [47] [48] due to free from side-chains on the latter molecules. The D-mannose-specific interaction between the extra-cellular bacterial polysaccharide xanthan and typical galactomannan and glucomannan components of the plant cell wall suggest a role in the host-pathogen relationship because Xanthomonas campestris is one of the plant pathogen bacteria.

We also discussed the molecular origin for the thermal stability of non-gelling polysaccharides, such as, welan [30], rhamsan [31] [32], S-657 gum [33], S-88 gum [49], schizophyllan [50], and rice amylopectin [20]-[22]. The structures of these polysaccharides are similar to those of the gelling polysaccharides, gellan gum, curdlan and amylose. The thermal stability of the polysaccharides as described above is attributed to intramolecular associations (hydrogen bonding and van der Waals forces of attraction), where methyl and hydroxyl groups, and hemiacetal oxygen atom contributed.

We observed that there are some basic rules in the gel-formation processes of polysaccharides [19] [29]. The gelation occurs because of the formation of intra- and inter-molecular associations, where hemiacetal oxygen, hydroxyl or methyl groups of the sugar residues of the polysaccharides contribute to hydrogen bonding or van der Waals forces of attraction. The sulfuric acid and carboxyl groups of the sugar residues of certain acidic polysaccharides, e.g., $\kappa$-carrageenan, $l$-carrageenan, gellan gum, alginic acid, and deacetylated rhamsan gum also participated in intra- and/or intermolecular associations through univalent or divalent cations that have ionic bonding or electrostatic forces of attraction.

\subsection{Gelation Mechanism of $\kappa$-Carrageenan}

$\kappa$-Carrageenan is well known for its gel-forming property [5] [7] [51]-[56]. The polysaccharide consists of alternating disaccharide units of $O$-3-linked $\beta$-D-galactopyranosyl 4-sulfate and $O$-4-linked 3,6-anhydro-D-galactopyranosyl residues (Figure 1) and used in food, cosmetic and other industries. A very large increase in elastic modulus was observed on addition of $13.5 \mathrm{mM} \mathrm{KCl}$ to a $0.2 \%$ solution of $\kappa$-carrageenan [5]. The tendency of the curve showing the elastic modulus for an increase in temperature up to $10^{\circ} \mathrm{C}$ suggested that intra- and inter-molecular association co-involved. However, least elastic modulus was observed on addition of $16.6 \mathrm{mM}$ $\mathrm{NaCl}$. $\kappa$-Carrageenan is a cation-selective binding polymer [5] [51]-[56], which gels in the presence of large-site univalent cations, such as $\mathrm{K}^{+}$(van der Waals rad. $\left.275 \mathrm{pm}\right), \mathrm{Rb}^{+}(303 \mathrm{pm})$ and $\mathrm{Cs}^{+}(343 \mathrm{pm})$, but does not do so in the presence of small cations, i.e., $\mathrm{Na}^{+}(227 \mathrm{pm})$ and $\mathrm{Li}^{+}(182 \mathrm{pm})$. This characteristic behavior was confirmed for the potassium and sodium salts of $\kappa$-carrageenan in our study [5] [7]. The cations having a negative JonesDole B coefficient of viscosity are structure disordering and show negative hydration of water molecules, whereas the cation having a positive coefficient are structure ordering and show positive hydration [57] [58]. The $\mathrm{K}^{+}$ ion has a negative $\mathrm{B}$ coefficient whereas the $\mathrm{Na}^{+}$ion has a positive one. Furthermore, the 3,6-anhydro- $\alpha$-D-galactose residues affect the hydrophobic character of the polymer by controlling its solubility. At low temperature, in the presence of ionic bonding between the sulfate and a large cation, it would be attracted to the adjacent 3,6-anhydro oxygen atom by the electrostatic forces of attraction.

On this basis, we proposed an intramolecular $\mathrm{K}^{+}$-bridge on $\kappa$-carrageenan molecule between sulfate oxygen of C-4 which oriented at axial configuration of D-galactopyranosyl residue and 3,6-ring oxygen of 3,6-anhydro- $\alpha$ D-galactopyranosyl residue, as illustrated in Figure 2 [5]. The bridge built up at low temperature, owing to the decrease in kinetic energy and Brownian motion of the molecular chains and solvent (water), but dissociation take place at temperature range of $10^{\circ} \mathrm{C}-25^{\circ} \mathrm{C}$, which was estimated to be a transition temperature, owing to an increase in kinetic energy and Brownian motion. The intramolecular bridge does not take place in the presence of $\mathrm{Na}^{+}$ion in spite of the ionic bonding still being present at low temperature, because the radius of the ion is too small for an association with the ring oxygen group and the presence of too much hydration prevents an electrostatic forces of attraction being involved in the bridge. The cation-specific intramolecular bridge, which was preferentially placed to make polysaccharide molecule rigid, was the first demonstration of this effect at a molecular level [5]. The bridge is likely to be established in view point of the flexibility of the $\alpha$-D- $(1 \rightarrow 3)$ linkage between the 3,6-anhydro-D-galactopyranosyl and the D-galactopyranosyl-4-sulfate, the sulfate group of which is oriented at axial configuration and corresponds to a rod-like and less extended structure.

On the basis of the intramolecular cation-bridge proposed, a model of intermolecular association of the $\kappa$ carrageenan molecules was developed [6] [7]. As the kinetic energy and Brownian motion of the molecules and 


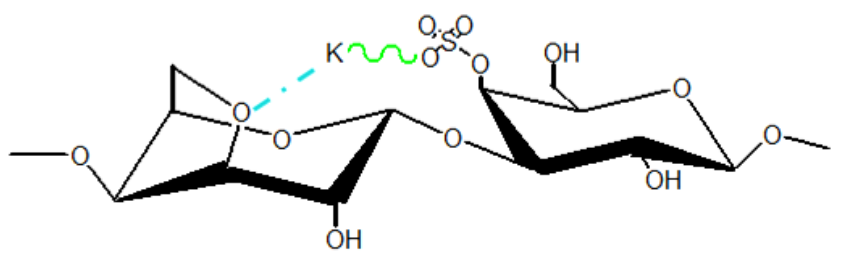

Figure 2. Intramolecular cation selective bridge in $\kappa$-carrageenan inaquesou solution at low temperature: (ionic bonding and $(-=)$ electrostatic forces of attraction.

solvent decreases at low temperature, many intramolecular cation-bridges serve to keep rigid the chains, which results in an intermolecular association. This suggests that the ring oxygen groups play the same role in the intermolecular association as do the sulfate groups. Thus, we proposed an intermolecular association between sulfuric acid groups on different molecules with electrostatic forces of attraction, as presented in Figure 3 [6] [7]. The transition temperature was observed at $25^{\circ} \mathrm{C}$ where inter- and intramolecular associations dissociated above the temperature.

\subsection{Gelation Mechanism of $\iota$-Carrageenan}

${ }_{l}$-Carrageenan is a more highly sulfated galactan and is also most characterized polysaccharide [59]-[65]. The polymer consist of $O$-3-linked $\beta$-D-galactopyranosyl 4-sulfate and $O$-4-linked 3,6-anhydro- $\alpha$-D-galactopyranosyl 2-sulfate residues (Figure 1). The polysaccharide is used in food, cosmetic and other industries as in $\kappa$ carrageenan. Gelation occurred for Ca-salt of $l$-carrageenan on cooling, but did not for $\mathrm{K}$ or Na salts [8]. The transition temperature was observed at $45^{\circ} \mathrm{C}$. Furthermore, a little increase in elastic modulus was observed during increasing temperature up to $10^{\circ} \mathrm{C}$ as same as in $\kappa$-carrageenan solution [5]. We concluded that the Ca salt of l-carrageenan involves an intramolecular association through Ca cation with ionic bonding between sulfate oxygens, as illustrated in Figure 4 [8]. Each cation is co-ordinated to two sulfate groups by ionic bonding. Since ionic bonding is stronger than electrostatic forces of attraction, the molecular chain become rigid even at room temperature and, therefore, intermolecular associations take place.

Thus intramolecular Ca-bridge proposed was developed into an intermolecular association mechanism of the Ca salt of $l$-carrageenan [8]. The intermolecular Ca-bridges may take place on different molecules having electrostatic forces of attraction (Figure 5). The intra- and inter-molecular cation bridges of the Ca-salt of $l$-carrageenan differs essentially from that of the K-salt of $\kappa$-carrageenan, because the former consists of double ionic forces and electrostatic forces of attractions, whereas the latter consists of single bonding and triple electrostatic forces of attraction. These different mechanism provide an explanation for the conformational transition of $l^{-}$ and $k$-carrageenan in aqueous solution. Since ionic bonding is stronger than electrostatic forces of attraction, the molecular chain can keep rigid even at intermediate temperature $\left(45^{\circ} \mathrm{C}\right)$ which was conformational transition temperature [8]. Intra- and inter-molecular Ca-bridges dissociate above the transition temperature.

\subsection{Gelation Mechanism of Agarose (Agar)}

Agarose is the major gelling constituent of agar extracted from red seaweeds. The polymer forms strong gelling ability [10] [66] [67] and is used in the separation, purification, and characterization of biomacromolecules in such techniques as gel filtration, affinity chromatography [68], hydrophobic chromatography [69] and electrophoresis. The polymer is currently attempted to use in pharmaceutical [1], drug delivery and tissue engineering [3] as a gelling agent. It consists of a copolymer alternating $O$-3-linked $\beta$-D-galactopyranosyl and $O$-4-linked 3,6 -anhydro- $\alpha$-L-galactopyranosyl residues, the structure of which is similar to that of $\kappa$-carrageenan and $l$-carrageenan, except for the sulfate content and L-configuration (Figure 1).

Gelation occurred at a concentration of $0.08 \%$ at low temperature $\left(0^{\circ} \mathrm{C}\right)$ and stayed constant during increase in temperature up to $60^{\circ} \mathrm{C}$ in the presence of $0.1 \%$ of salt $\left(\mathrm{NaCl}, \mathrm{KCl}, \mathrm{CaCl}_{2}\right.$, or $\left.\mathrm{MgCl}_{2}\right)$, then decreased rapidly with further increase in temperature [10]. This might be caused by salting-out effect which might take place because of the rigidity of the molecular chains of agarose through the formation of intra- and inter-molecular association, resulting from the tetrahedral distribution of anhydro- $\alpha$-L-galactosyl residues and water molecules. However, the least elastic modulus was observed in the presence of urea (4.0 M). Thus, we proposed an intra- 


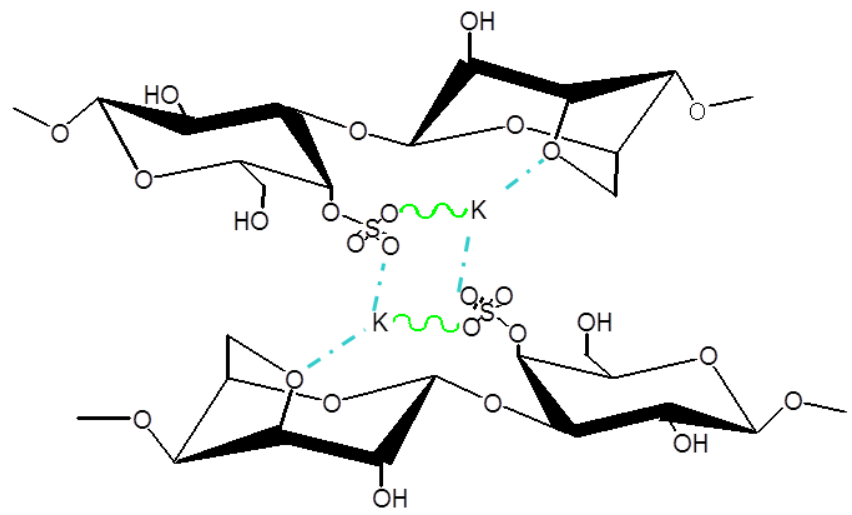

Figure 3. Gelation mechanism of $\kappa$-carrageenan: (onic bonding and ( $-{ }^{-}-$) electrostatic forces of attraction.

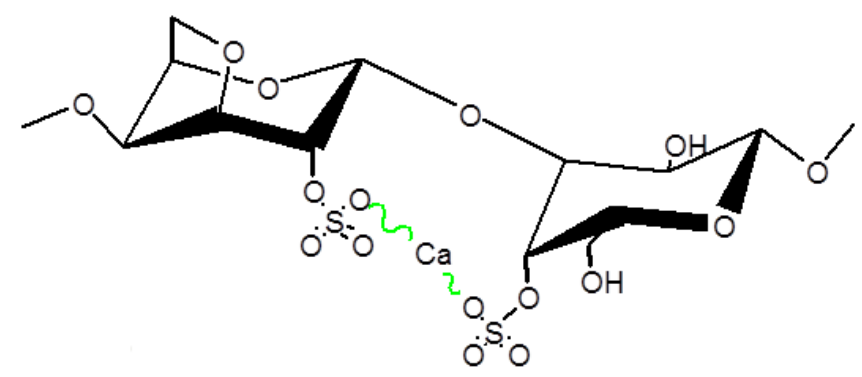

Figure 4. Intramolecular $\mathrm{Ca}^{2+}$ bridge with ionic bonding ( in $l$-carrageenan molecule.



Figure 5. Gelation mechanism of $l$-carrageenan: ( bonding and ( - - - ) electrostatic forces of attraction.

molecular hydrogen bonding between $\mathrm{OH}-4$ which oriented at axial configuration of the $\beta$-D-galactopyranosyl and the adjacent hemiacetal oxygen atom of the 3,6-anhydro- $\alpha$-L-galacctopyranosyl residues (Figure 6) [9]. The 3,6-anhydro-L-galactopyranosyl residue is a cage-like sugar that contributes by stabilizing the proposed intramolecular hydrogen bonding, even at high temperature $>60^{\circ} \mathrm{C}$. Intermolecular hydrogen bonding was also proposed between the ring $O-3,6$-atom and the $\mathrm{OH}-2$ which oriented at axial configuration of 3,6-anhydro- $\alpha$-L-galactopyranosyl residues on different molecules (Figure 7) [10]. This intermolecular hydrogen bonding results from the cage effect of the 3,6-anhydro- $\alpha$-L-galactopyranosyl residues which adopt a tetrahedral distribution and therefore attract not only each other, but also water molecules with hydrogen bonding. The mode of intra- and intermolecular hydrogen bonding of agarose molecules has been supported by ${ }^{1} \mathrm{H}-$ and ${ }^{13} \mathrm{C}-\mathrm{NMR}$ spectroscopy [70]. 


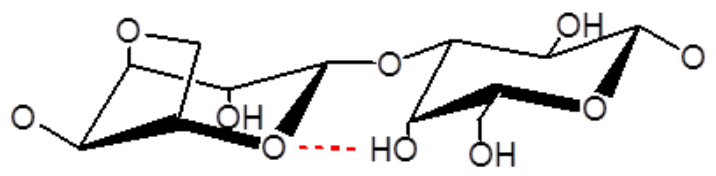

Figure 6. Intramolecular hydrogen bonding $(---)$ in agarose molecule.

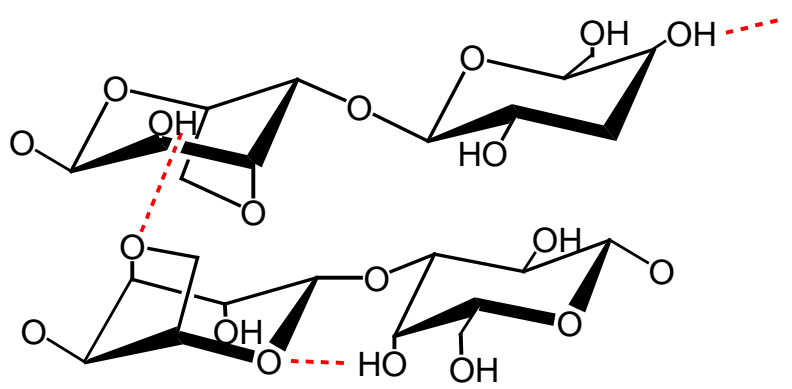

Figure 7. Gelation mechanism of agarose. $(---)$ : Hydrogen bonding.

Although agarose is a diastereomeric derivative of $\kappa$-carrageenan and $l$-carrageenan, it shows an intramolecular association similar to that of the $\kappa$ - and $l$-carrageenan. The role of such polysaccharides in biological tissues seems to be the cohesion, the retention of water and salts, and the physical organization. Thus, the gelation mechanisms for these polysaccharides, proposed may be useful for further developments of not only industrial applications, but also for an understanding of the biological functions.

\subsection{Molecular Origin for Rheological Characteristics of Gellan Family of Polysaccharides, Gellan, Welan, Rhamsan, S-657 Gum and Deacetylated Rhamsan Gum}

\subsubsection{Gelation Mechanism of Gellan Gum}

Gellan gum is a bacterial polysaccharide produced by Sphingomonas elodea and has potential industrial applications as a gelling agent alternate to agar (agarose) and carrageenans in food industry and biotechnology [71] [72]. The primary structure of the polysaccharide consists of tetrasaccharide repeating units (Figure 1), 3 )- $\beta$-DGlcp-( $1 \rightarrow 4)-\beta$-D-GlcpUA-( $1 \rightarrow 4)-\beta$-D-Glcp-( $1 \rightarrow 4)-\alpha$-L-Rhap-(1 [73] [74]. The polysaccharide contains $6 \%$ of $O$-acetyl and L-glycerate groups at C-6 and C-2 of $\rightarrow 3)-\beta$-D-Glcp-(1 [75]. Under suitable condition, the native gellan gum forms weak gels [12], however, it forms stiff, brittle gels on deacylation [11] as in agarose. The physicochemical investigations for deacylated gellan gum were done by many workers [76]-[78]. A tertiary structure of the polymer was reported to consist of two identical left-handed, 3-fold double helices in the solid state [78] similar to that of agarose.

A gelation occurred in gellan gum $\left(\mathrm{K}^{+}\right.$salt) concentration at $0.8 \%$ upon cooling and transition temperature was observed at $15^{\circ} \mathrm{C}$ and $20^{\circ} \mathrm{C}$ at $0.8 \%$ and $1.0 \%$ [11]. In the presence of $\mathrm{CaCl}_{2}(6.8 \mathrm{mM})$, very large increase in elastic modulus was observed in $0.2 \%$ gellan solution and kept a constant value during increase in temperature to $80^{\circ} \mathrm{C}$, which was estimated to be a transition temperature, then decreased rapidly. It meant that the thermal stable gelation occurred. However, the polymer showed a little increase in elastic modulus even at $0.4 \%$ in the presence of $\mathrm{KCl}(13.5 \mathrm{mM})$, but stayed at low value with addition of $\mathrm{NaCl}(16.6 \mathrm{mM})$. On the contrary, an increase in elastic modulus occurred in the presence of urea $(4.0 \mathrm{M})$ which was well known as a hydrogen bonding breaker. The result suggested that hydrophobic interaction took place in gel formation process [12].

On the basis of the experimental results, we proposed a gelation mechanism of gellan gum as shown in Figure 8. The intramolecular hydrogen bonding might take place between OH-4 of the D-glucopyranosyl residue and the adjacent hemiacetal oxygen atom of the L-rhamnopyranosyl residue and between $\mathrm{OH}-3$ of the D-glucopyranosyl residue and the adjacent hemiacetal oxyten atom of the D-glucuropyranosyl residue, to make gellan gum molecule rigid. The thermal stable gelation might be attributed to contributing methyl group of L-rhamnopyranosyl residue [11] [12]. Thus, we proposed an intermolecular van der Waals interaction between the methyl group which oriented at axial configuration of the L-rhamnopyranosyl residue due to taking ${ }^{4} \mathrm{C}_{1}$ py- 


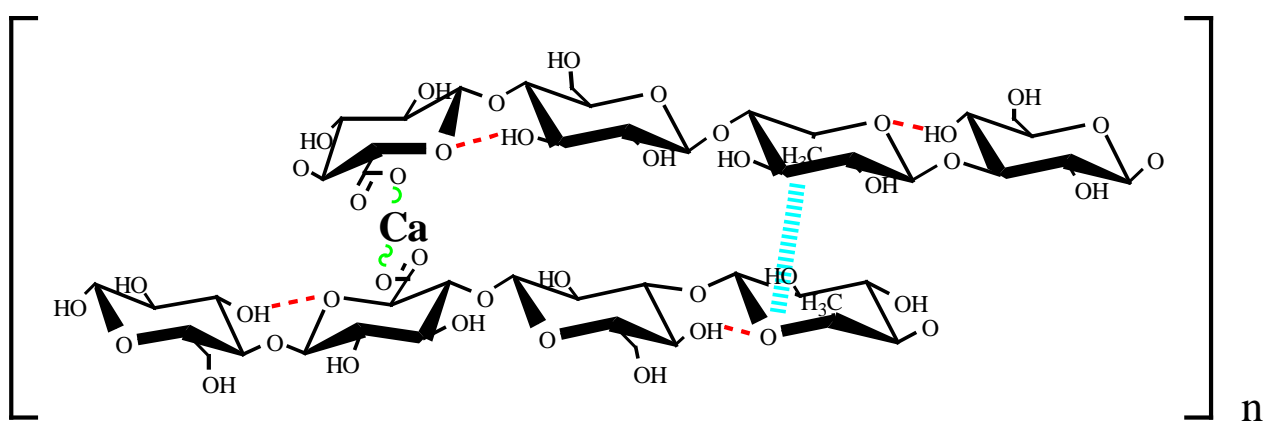

Figure 8. Gelation mechanism of gellan gum in aqueouss solution. Association; - - - - Hydrogen bonding;

ranose-ring conformation and the hemiacetal oxygen atom of the L-rhamnopyranosyl residue on different molecules. In the presence of $\mathrm{Ca}^{2+}$ ions, an intermolecular $\mathrm{Ca}^{2+}$ bridge also took place with ionic bonding between carboxyl oxygen atoms of D-glucuropyranosyl residues on different molecules. The intermolecular van der Waals forces of attraction and ionic bonding were stable even at high temperature $\left(80^{\circ} \mathrm{C}\right)$.

\subsubsection{Molecular Origin for Thermal Stability of Welan Gum}

Welan gum is produced by Alcaligenes ATCC 31555, and is non-gel forming, but gives a thermostable highly viscous solutions at a high temperature $>100^{\circ} \mathrm{C}$ [71] [72] [79] [80]. The main chain of welan gum consists of tetrasaccharide repeating units as in gellan gum and every D-glucopyranosyl residue next to the D-glucuropyranosyl residue is substituted at $O-3$ by either $\alpha$-L-rhamnopyranosylot $\alpha$-L-mannopyranosyl residue in the ratio of $2: 1[81]$.

The elastic modulus was scarcely changeable with increasing temperature even in $1.0 \%$ solution at high temperature $\left(80^{\circ} \mathrm{C}\right)$ [30]. A little decrease of elastic modulus was observed with addition of $\mathrm{CaCl}_{2}(6.8 \mathrm{mM})$, indicating that side-chains of welan gum molecules prevent the formation of intermolecular $\mathrm{Ca}^{2+}$ bridges. Thus, intramoleclar associations between the $\mathrm{OH}-4$ of the D-glucopyranosyl residue and the adjacent hemiacetal oxygen atom of the L-rhamnopyranosyl residue with hydrogen bonding, and between the methyl group of the L-rhamnopyranosyl residue which oriented at equatorial configuration because of taking ${ }^{1} \mathrm{C}_{4}$ pyranose-ring conformation and the adjacent hemiacetal oxygen atom of the D-glucopyranosyl residue with van der Waals forces of attraction were proposed (Figure 9) [30]. The intramolecular associations contributed to the thermal stability of the polysaccharide in aqueous solutions.

\subsubsection{Molecular Origin for Thermal Stability of Rhamsan Gum}

Rhamsan gum is produced by Alcaligenes ATCC 31961, and non-gel forming but gives a thermostable, highly viscous solution even at high temperature $>100^{\circ} \mathrm{C}$ [71] [72]. The main chain of the rhamsan gum consists of tetrasaccharide repeating units as in gellan gum and every D-glucopyranosyl residue next to the L-rhamnopyranosyl residue is substituted at C-6 by $\beta$-D-glucopyranosyl- $(1 \rightarrow 6)$ - $\alpha$-D-glucopyranosyl disaccharide side-chains [82].

A very large elastic modulus was observed at concentration above $0.3 \%$ and kept a constant value with increasing temperature [31] [32]. A weak sigmoid curve was shown at $1.0 \%$ concentration the phenomenon of which was similar to that of xanthan gum [34] [38] [39]. The elastic modulus of rhamsan gum (0.2\%) showed almost the same values with addition of $\mathrm{CaCl}_{2}(6.8 \mathrm{mM})$ during increase in temperature in comparison with polysaccharide alone. Thus, intramoleclar associations between the $\mathrm{OH}-4$ of the D-glucopyranosyl residue and the adjacent hemiacetal oxygen atom of the L-rhamnopyranosyl residue with hydrogen bonding, and between the methyl group of the L-rhamnopyranosyl residue which oriented at equatorial configuration and the adjacent hemiacetal oxygen atom of the D-glucopyranosyl residue with van der Waasl forces of attraction were proposed (Figure 10) [31] [32]. The intramolecular association contributed to the thermal stability of the polysaccharide in aqueous solutions.

\subsubsection{Molecular Origin for Thermal Stability of S-657 Gum}

The S-657 gum produced by Xanthomonas ATCC 53159 is non-gelling but gives a thermostable, highly viscous 


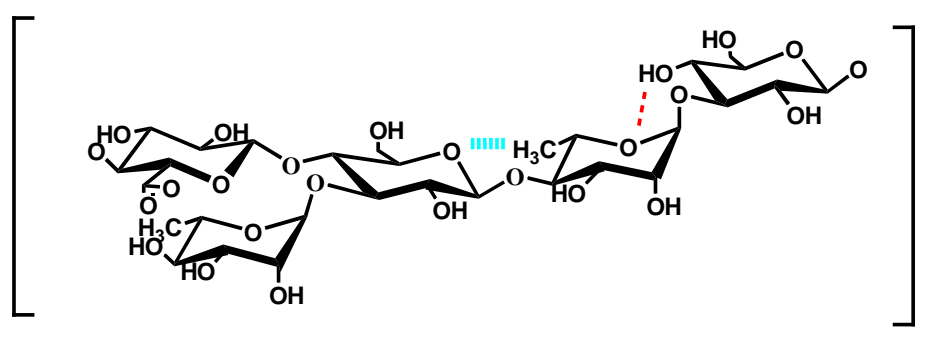

Figure 9. Intramolecular associations in welan gum. Associations:,--- , Hydrogen bonding; $/ / / /$, van der Waals forces of attraction.

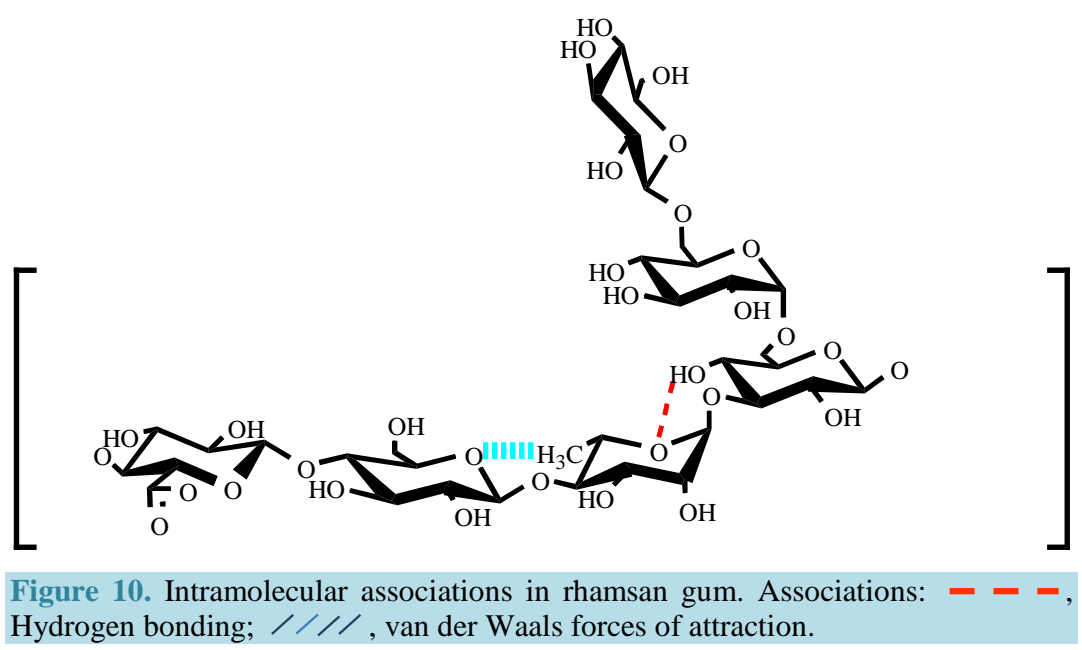

solution even at high temperatures [71] [72] [83]. The main chain of the rhamsan gum consists of tetrasaccharide repeating units as in gellan gum and every D-glucopyranosyl residue next to the D-glucuropyranosyl residue is substituted at C-3 by $\alpha$-L-rhamnopyranosyl-( $1 \rightarrow 4)$ - $\alpha$-L-rhamnopyranosyl disaccharide side-chains [84].

Gelation did not occur even at $1.0 \%$ and at low temperature $\left(0^{\circ} \mathrm{C}\right)$. The elastic modulus showed a sigmoid curve with increasing temperature. Almost same elastic modulus was observed during increase in temperature in the presence of $\mathrm{CaCl}_{2}(6.8 \mathrm{mM})$. Thus, intramolecular associations between the $\mathrm{OH}-4$ of the D-glucopyranosyl residue and the adjacent hemiacetal oxygen atom of the L-rhamnopyranosyl residue with hydrogen bonding, and between the methyl group of the L-rhamnopyranosyl residue which oriented at equatorial configuration and the adjacent hemiacetal oxygen atom of the D-glucopyranosyl residue with van der Waals forces of attraction were proposed (Figure 11) [33]. The intramolecular associations contributed to the thermal stability of the polysaccharide in aqueous solutions.

\subsubsection{Gelation Mechanism of Deacetylated Rhamsan Gum}

The rhamsan gum contains one $O$-acetyl group per tetrasaccharide repeating units [81], and is non gelling polysaccharide, as mentioned above. However, after deacetylation, the polymer formed gel above $0.3 \%$ at low temperature $\left(0^{\circ} \mathrm{C}\right)$ [18]. A gelation occurred in $0.2 \%$ deacetylated rhamsan gum solution with addition of $\mathrm{CaCl}_{2}(6.8$ $\mathrm{mM}$ ). The elastic modulus kept a constant value during increase in temperature up to $60^{\circ} \mathrm{C}$, which was estimated a transition temperature, then decreased rapidly. The elastic modulus of deacetylated rhamsan gum $(0.5 \%)$ increased a little with addition of urea $(4.0 \mathrm{M})$ at low temperature and kept constant values during increase in temperature and decreased rapidly when the temperature reached $50^{\circ} \mathrm{C}$, which was estimated transition temperature. As mentioned above, elastic modulus of gellan gum solution also increased on addition of urea (4 M) at low temperature, but it decreased rapidly after temperature reached $15^{\circ} \mathrm{C}$.

Rheological properties of deacetylated rhamsan gum differed from those of native polymer [18], but agreed with those of gellan gum [11] [12]. Thus, we proposed intramolecular and intermolecular associations, as shown in Figure 12 [18], which was similar to those of gellan gum (Figure 8). The intramolecular hydrogen bonding might take place between OH-4 group of the D-glucopyranosyl residue and adjacent hemicetal oxygen atom of 




Figure 11. Intramolecular associations in S-657 gum. Associations: --- , Hydrogen bonding; $/ / / /$, van der Waals forces of attraction.



Figure 12. Gelation mechanism of deacetylated rhamsan gum. Associations: $-{ }^{-}-$, Hydrogen bonding; //// , van der Waals forces of attraction;

the L-rhamnopyranosyl residue, and between OH-3 of the D-glucopyranosyl residue and adjacent hemiacetal oxygen atom of the D-glucuropyranosyl residue, to make the polymer rigid. The intermolecular van der Waals forces of attraction might take place between the methyl group and counter hemiacetal oxygen atom of Lrhamnopyranosyl residues on different molecules. In the presence of $\mathrm{CaCl}_{2}$, an intermolecular $\mathrm{Ca}^{2+}$ bridge, as in l-carrageenan [8], gellan gum [11] [12] and alginate [16] [17], might also take place with ionic bonding. Although the L-rhamnopyranosyl residues in part of native rhamsan gum adopt a ${ }^{1} \mathrm{C}_{4}$ pyranose-ring conformation, they exit in a ${ }^{4} C_{1}$ pyuranose-ring conformation in deacetylated rhamsan gum [18]. Such a conformational change of the L-rhamnosyl residue was confirmed by ${ }^{1} \mathrm{H}-\mathrm{NMR}$ spectroscopy [18]. The gelation mechanism of deacetylated rhamsan gum was proposed, as shown in Figure 12 [18].

\section{Principle of Polysaccharide Gels}

The $\mathrm{H}_{2} \mathrm{O}$ molecule can participate in four hydrogen bonds, two of them involving the two hydrogens of the molecule and the lone pair of electrons of the oxygen and hydrogens of two neighboring molecules. This tetrahedrally directed bonding is involved in the crystal structure of ice, which is the same as the arrangement of the gelling waters in polysaccharide solution. At a minimum concentration of $0.1 \%-1.0 \%(\mathrm{~W} / \mathrm{V})$ in water $(99.9 \%$ $99.0 \%$ ), the gelling polysaccharides mentioned above changed into an ice-like structure with the formation of hydrogen bonding between the polysaccharides and water, and between water molecules, which subsequently 
resulting in gelation even at room temperature. Such dramatic changes from liquid into gels have generally been understood at the molecular level [19] [29].

We discuss herein the role of polysaccharides for an example agarose in gel-formation processes in water. On the basis of the discussions mentioned above, the agarose molecules play a dominant role in the center of tetrahedral cavities (cages) that are occupied by water molecules. This arrangement is similar to a tetrahedral ice-like structure and should lead to a cooperative effect. This effect stabilizes extended regions of the ice-like water that is hydrogen bonding on the surface of the polymer molecules. On the surface of the polymer molecules, ring oxygen and hydroxyl groups participate in hydrogen bonding with water molecules, and more extended ice-like hydrogen bonding with water molecules achieved to form a cluster, as shown in Figure 13. Namely, agarose gel



Figure 13. Gelling agarose in water molecules. Red dotted lines represent hydrogen bonding. 
formation corresponds to a total of the clusters. The intra- and inter-molecular associations result in cage effect, which lead to the lowest energy state of electrons of the lone pairs of 3,6-anhydro-oxygen and hemiacetal oxygen atoms. The conformation adopts a tetrahedral distribution, and therefore, attracts not only each other but also water molecules due to hydrogen bonding. The intra- and inter-molecular hydrogen bonding of agarose molecules results in gelation up to a high temperature $\left(60^{\circ} \mathrm{C}\right)$, and their periphery is surrounded by hydrophobic carbon and hydrogen atoms, which are required to play a role in the hydrophobic effect. This hydrophobic effect leads to hydrogen bonding that easily occurs within water molecules because of decrease in entropy. Therefore, hydrogen bonding also takes place easily with water molecules on the outside of the polymer helices (Figure 13).

Accordingly, the gel-forming polysaccharides molecules, as mentioned above, may adopt single, double, or multi-stranded conformations involving intra- and inter-molecular associations and play a role in the center of the tetrahedral cavities leading and stabilizing the extended regions of ice-like water molecules with hydrogen bonding, and resulting in gelation.

\section{Conclusions}

In gel-formation processes of biopolymer, polysaccharides, cage and hydrophobic effect play thermal dynamically dominant role. The former effect is observed in daily life: Water molecules at low temperature around $0^{\circ} \mathrm{C}$ immediately changed into ice crystals upon the addition of some contaminant such as small dust particles which lead to the lowest energy state of electrons, between water molecules through tetrahedrally directed hydrogen bonding. Furthermore, an amylose solution at a concentration of $1.0 \%(\mathrm{~W} / \mathrm{V})$, changed into a gel immediately under weak mechanical stimulation (cage effect) [13] [14]. The latter effect is also observed: A droplet of water forms a spherical shape where tetrahedrally directed hydrogen bonding takes place, minimizing contact with the hydrophobic plant leaf surface. This hydrophobic effect leads to hydrogen bonding that easily occurs within water molecules because of a decrease in entropy. Such effect, cage and hydrophobic, may also play role in proteins, synthesized polymers and sugar-fatty acid complex gel formation processes. Specifically, hydrophobic effect may play in organo-gel formation in organic solvents with van der Waals forces of attraction.

Though many investigations the gelling properties of the polysaccharides have been undertaken to elucidate the structure-function relationship, no other researchers have established mechanism at the molecular level. There is reasonable consistency in our investigations. Thus, the rheological analysis is one of the significant methods for understanding the structure-function relationship of polysaccharides in aqueous media. We expect that the era of polysaccharides gels will be arriving in the 21st century, being applied not only from the food industry, but also in a variety of products, such as cosmetics, pharmaceuticals, chemicals, drug delivery, tissue engineering, and other industries.

\section{Acknowledgements}

Author wishes to thank the late Professor Danji Nomura who was my supervisor in Doctoral Course in Food Science and Technology of Kyushu University for his encouraging me to talk many stories about Nobel Laureate Hans von Euler-Chelpin because Professor Nomura had studied in Laboratory of Professor Euler-Chelpin, University of Stockholm, Sweden in 1959-1960.

Author has also heard lot about Emil Fischer and van't Hoff because Both Nobel Laureates were professors of Hans von Euler-Chelpin in Berlin University, Germany. Professor van't Hoff is well known as a man who created stereo chemistry by finding out the carbon atom involves tetrahedral conformation, and relationship between stereo isomers and their optical activity. Professor Emil Fischer is also well known as a man who established stereo chemistry by elucidating chemical structure of D-glucose, D-galactose, D-mannose and D-fructose.

Author was a lucky student because Professor Danji Nomura, Hans von Euler-Chelpin, van’t Hoff and Emil Fischer have led me on the road to the structure-function relationship of the polysaccharides when I was listening to the first movement of Piano Sonata No. 14 Op. 27-2 "Moon Light" composed by Ludwig van Beethoven on July 16th in 1973.

\section{References}

[1] Rinaudo, M. (2008) Main Properties and Current Applications of Some Polysaccharides as Biomaterials. Polymer International, 57, 397-430. http://dx.doi.org/10.1002/pi.2378

[2] Laurienzo, P. (2010) Marine Polysaccharides in Pharmaceutical Applications. Marine Drug, 8, 2435-2465. 
http://dx.doi.org/10.3390/md8092435

[3] Lee, K.Y. and Mooney, D.J. (2001) Hydrogels for Tissue Engineering. Chemical Reviews, 101, 1869-1879.

[4] Tako, M., Nagahama, T. and Nomura, D. (1977) Flow Characteristics of Viscous Polysaccharide Produced by Coryneform Bacteria Strain C-8. Nippon Nogeikagaku Kaishi, 51, 397-403. http://dx.doi.org/10.1271/nogeikagaku1924.51.6_397

[5] Tako, M. and Nakamura, S. (1986) Indicative Evidence for a Conformational Transition in $\kappa$-Carrageenan from Studies of Viscosity-Shear Rate Dependence. Carbohydrate Research, 155, 200-205. http://dx.doi.org/10.1016/S0008-6215(00)90146-0

[6] Tako, M. and Nakamura, S. (1986) Synergistic Interaction between $\kappa$-Carrageenan and Locust Bean Gum in Aqueous Media. Agricultural Biological Chemistry, 50, 2817-2822. http://dx.doi.org/10.1271/bbb1961.50.2817

[7] Qi, Z.-Q., Tako, M. and Toyama, S. (1998) Molecular Origin for Rheological Characteristics of $\kappa$-Carrageenan Isolated from Ibaranori (Hypnea charoides). Journal of Applied Glycoscience, 44, 331-336.

[8] Tako, M., Nakamura, S. and Kohda, Y. (1987) Indicative Evidence for a Conformational Transition in $l$-Carrageenan. Carbohydrate Research, 161, 247-253. http://dx.doi.org/10.1016/S0008-6215(00)90081-8

[9] Lin, H.-L., Tako, M. and Hongo, F. (2000). Molecular Origin for Rheological Characteristics of $\imath$-Carrageenan Isolated from Togekirinsai (Eucheumaserra). Food Science and Technology Research, 43, 493-498.

[10] Tako, M. and Nakamura, S. (1988) Gelation Mechanism of Agarose. Carbohydrate Research, 180, 277-284. http://dx.doi.org/10.1016/0008-6215(88)80084-3

[11] Tako, M., Sakae, A. and Nakamura, S. (1989) Rheological Properties of Gellan Gum in Aqueous Media. Agricultural Biological Chemistry, 53, 771-776. http://dx.doi.org/10.1271/bbb1961.53.771

[12] Tako, M., Teruya, T., Tamaki, Y. and Konishi, T. (2009) Molecular Origin for Rheological Characteristics of Native Gellan Gum. Colloid and Polymer Science, 287, 1445-1454. http://dx.doi.org/10.1007/s00396-009-2112-2

[13] Tako, M. and Hizukuri, S. (1995) Evidence for Conformational Transition in Amylose. Journal of Carbohydrate Chemistry, 14, 613-622. http://dx.doi.org/10.1080/07328309508005362

[14] Tamaki, Y., Konishi, T. and Tako, M. (2011) Gelation and Retrogradation Mechanism of Wheat Amylose. Materials, 4, 1763-1775. http://dx.doi.org/10.3390/ma4101763

[15] Tako, M. and Hanashiro, I. (1997) Evidence for a Conformational Transition in Curdlan. Polymer Gels \& Networks, 5, 241-250. http://dx.doi.org/10.1016/S0966-7822(96)00036-6

[16] Tako, M. and Kohda, Y. (1997) Calcium Induced Association Characteristics of Alginate. Journal of Applied Glycoscience, 44, 153-159.

[17] Teruya, T., Tamaki, Y., Konishi, T. and Tako, M. (2010) Rheological Characteristics of Alginate Isolated from Commercially Cultured Nemacystus decipiens (Itomozuku). Journal of Applied Glycoscience, 57, 7-12. http://dx.doi.org/10.5458/jag.57.7

[18] Tako, M., Tohma, S., Taira, T. and Ishihara, M. (2003) Gelation Mechanism of Deacetylated Rhamsan Gum. Carbohydrate Polymers, 54, 279-285. http://dx.doi.org/10.1016/S0144-8617(03)00029-8

[19] Tako, M. (2000) Structural Principles of Polysaccharide Gels. Journal of Applied Glycoscience, 47, 49-53. http://dx.doi.org/10.5458/jag.47.49

[20] Tako, M. and Hizukuri, S. (1997) Molecular Origin for Thermal Stability of Rice Amylopectin. Journal of Carbohydrate Chemistry, 16, 655-666.

[21] Tako, M. (1999) Molecular Origin for Thermal Stability of Waxy Rice Starch. Starch/Starke, 48, 414-417.

[22] Tako, M. and Hizukuri, S. (2000) Molecular Origin for Thermal Stability of Koshihikari Rice Amylopectin. Food Research International, 33, 35-40. http://dx.doi.org/10.1016/S0963-9969(00)00021-1

[23] Tako, M. and Hizukuri, S. (1999) Gelatinization Mechanism of Rice Starch. Journal of Carbohydrate Chemistry, 18, 573-584. http://dx.doi.org/10.1080/07328309908544020

[24] Tako, M. (2000) Gelatinization Characteristics of Rice Starch. Journal of Applied Glycoscience, 47, 187-192. http://dx.doi.org/10.5458/jag.47.187

[25] Tako, M. and Hizukuri, S. (2003) Gelatinization Mechanism of Potato Starch. Carbohydrate Polymers, 48, $397-401$. http://dx.doi.org/10.1016/S0144-8617(01)00287-9

[26] Tako, M., Tamaki, Y., Konishi, T., Shibanuma, K., Hanashiro, I. and Takeda, Y. (2008) Gelatinization and Retrogradation Characteristics of Wheat (Rosella) Starch. Food Research International, 41, 797-802. http://dx.doi.org/10.1016/j.foodres.2008.07.002

[27] Tako, M., Tamaki, Y., Teruya, T., Konishi, T., Shibanuma, K., Hanashiro, I. and Takeda, Y. (2009) Gelatinization Characteristics of Halberd Wheat Starch. Starch/Starke, 61, 275-281. 
[28] Tako, M. and Hizukuri, S. (2000) Retrogradation Mechanism of Rice Starch. Cereal Chemistry, 77, 473-477.

[29] Tako, M., Tamaki, Y., Teruya, T. and Takeda, Y. (2014) The Principles of Starch Gelatinization and Retrogradation. Food Nutrition and Sciences, 5, 280-291. http://dx.doi.org/10.4236/fns.2014.53035

[30] Tako, M. and Kiriaki, M. (1990) Rheological Properties of Welan Gum in Aqueous Media. Agricultural Biological Chemistry, 54, 3079-3084. http://dx.doi.org/10.1271/bbb1961.54.3079

[31] Tako, M. (1993) Molecular Origin for Thermal Stability of Rhamsan Gum in Aqueous Media. Bioscience Biotechnology Biochemistry, 57, 1182-1184. http://dx.doi.org/10.1271/bbb.57.1182

[32] Tako, M. (1993) Molecular Origin for the Thermal Stability of Welan and Rhamsan Gum. In: Yalpani, M., Ed., Carbohydrate and Carbohydrate Polymers, ATL Press, Inc. Science and Publishers, Atlanta, 206-215.

[33] Tako, M. (1994) Molecular Origin for Thermal Stability of S-657 Polysaccharide Produced by Xanthomonas ATCC 53159. Polymer Gels \& Networks, 2, 91-104. http://dx.doi.org/10.1016/0966-7822(94)90029-9

[34] Tako, M., Nagahama, T. and Nomura, D. (1977) Non-Newtonian Behavior and Dynamic Viscoelasticity of Xanthan Gum. Nippon Nogeikagaku Kaishi, 51, 513-517. http://dx.doi.org/10.1271/nogeikagaku1924.51.8 513

[35] Tako, M. and Nakamura, S. (1984) Rheological Properties of Deacetylated Xanthan Gum in Aqueous Media. Agricultural Biological Chemistry, 48, 2987-2993. http://dx.doi.org/10.1271/bbb1961.48.2987

[36] Tako, M. and Nakamura, S. (1987) Rheological Properties of Ca Salt of Xanthan Gum in Aqueous Media. Agricultural Biological Chemistry, 51, 2919-2923. http://dx.doi.org/10.1271/bbb1961.51.2919

[37] Tako, M. and Nakamura, S. (1988) Rheological Properties of Depyruvated Xanthan Gum in Aqueous Media. Agricultural Biological Chemistry, 52, 1585-1586. http://dx.doi.org/10.1271/bbb1961.52.1585

[38] Tako, M. and Nakamura, S. (1989) Evidence for Intramolecular Associations in Xanthan Molecules in Aqueous Media. Agricultural Biological Chemistry, 53, 1941-1946. http://dx.doi.org/10.1271/bbb1961.53.1941

[39] Tako, M. (1992) Molecular Origin for Rheological Characteristics of Xanthan Gum. ACS Symposium Series, 489, 268281.

[40] Tako, M., Asato, A. and Nakamura, S. (1984) Rheological Aspects of Intermolecular Interaction between Xanthan and Locust Bean Gum in Aqueous Media. Agricultural Biological Chemistry, 48, 2995-3000. http://dx.doi.org/10.1271/bbb1961.48.2995

[41] Tako, M. and Nakamura, S. (1986) Synergistic Interaction between Xanthan and D-Galacto-D-Mannan. FEBS Letters, 204, 33-36.

[42] Tako, M. (1992) Synergistic Interaction between Xanthan and Galactomannan. Journal of Carbohydrate Chemistry, 10, 619-623. http://dx.doi.org/10.1080/07328309108543936

[43] Tako, M. and Nakamura, S. (1985) Synergistic Interaction between Xanthan and Guar Gum. Carbohydrate Research, 138, 207-213. http://dx.doi.org/10.1016/0008-6215(85)85104-1

[44] Tako, M. (1991) Synergistic Interaction between Xanthan and Tara-Bean Gum. Carbohydrate Polymers, 16, $227-239$. http://dx.doi.org/10.1016/0144-8617(91)90111-O

[45] Pakdee, P., Tako, M., Yokohari, T., Kinjyo, K., Hongo, F. and Yaga, S. (1995) Synergistic Interaction between Xanthan and Galactomannan Isolated from Leucaena leucocephala de Wit. Journal of Applied Glycoscience, 42, 105-113.

[46] Tako, M., Teruya, T., Tamaki, Y. and Ohkawa, K. (2010) Co-Gelation Mechanism of Xanthan and Galactomannan. Colloid and Polymer Science, 288, 1161-1166. http://dx.doi.org/10.1007/s00396-010-2242-6

[47] Tako, M. (1992) Synergistic Interaction between Xanthan and Konjac Glucomannan in Aqueous Media. Bioscience Biotechnology Biochemistry, 56, 1188-1192. http://dx.doi.org/10.1271/bbb.56.1188

[48] Tako, M. (1993) Binding Sites for D-Mannose-Specific Interaction between Xanthan and Galactomannan, and Glucomannan. Colloids and Surface B: Biointerfaces, 1, 125-131. http://dx.doi.org/10.1016/0927-7765(93)80043-X

[49] Tako, M. and Tamaki, H. (2005) Molecular Origin for the Thermal Stability of S-88 Gum Produced by Pseudomonas ATCC 31554. Polymer Journal, 37, 498-505. http://dx.doi.org/10.1295/polymj.37.498

[50] Tako, M. (1996) Molecular Origin for Thermal Stability of Schizophyllan. Polymer Gels \& Networks, 4, 303-313. http://dx.doi.org/10.1016/0966-7822(96)00016-0

[51] Rees, D.A. (1972) Shapely Polysaccharides. Biochemical Journal, 126, 257-273.

[52] Morris, V.J. and Chilvers, G.R. (1983) Rheological Studies of Specific Cation Forms of Kappa Carrageenan Gels. Carbohydrate Polymers, 3, 129-141. http://dx.doi.org/10.1016/0144-8617(83)90003-6

[53] Rochas, C. and Rinaudo, M. (1984) Mechanism of Gel Formation in $\kappa$-Carrageenan. Biopolymers, 23, 735-745. http://dx.doi.org/10.1002/bip.360230412

[54] Hagerth, A., Nilsson, S. and Sundelof, L.-O. (1999) Gel-Sol Transition in $\kappa$-Carrageenan Systems: Microviscosity of 
Hydrophobic Microdomains, Dynamic Rheology and Molecular Conformation. International Journal Biological Macromolecules, 26, 69-76. http://dx.doi.org/10.1016/S0141-8130(99)00065-3

[55] Chen, Y., Liao, M.-L. and Dunstan, D.E. (2002) The Rheology of $\mathrm{K}^{+}-\kappa$-Carrageenan as a Weak Gel. Carbohydrate Polymers, 50, 109-116. http://dx.doi.org/10.1016/S0144-8617(02)00009-7

[56] Kozbial, A. and Li, L. (2014) Study on the Friction of $\kappa$-Carrageenan in Air and Aqueous Environments. Materials Science Engineering: C, 36, 173-179. http://dx.doi.org/10.1016/j.msec.2013.12.003

[57] Frank, H.S. and Wen, W.Y. (1957) Ion-Solvent Interaction. Structural Aspects of Ion-Solvent Interaction in Aqueous Solutions: A Suggested Picture of Water Structure. Discussion Faraday Society, 24, 133-140. http://dx.doi.org/10.1039/df9572400133

[58] Kaminsky, M. (1957) Ion-Solvent Interaction and the Viscosity of Strong-Electrolyte Solutions. Discussion Faraday Society, 24, 171-179. http://dx.doi.org/10.1039/df9572400171

[59] Arnott, S., Scott, W.E., Rees, D.A. and McNab, C.G.A. (1974) i-Carrageenan: Molecular Structure and Packing of Polysaccharide Double Helices in Oriented Fibres of Divalent Cation Salts. Journal of Molecular Biology, 90, $253-267$. http://dx.doi.org/10.1016/0022-2836(74)90371-4

[60] Morris, E.R., Rees, D.A. and Robinson, G. (1980) Cation-Specific Aggregation of Carrageenan Helices: Domain Model of Polymer Gel Structure. Journal of Molecular Biology, 138, 349-362. http://dx.doi.org/10.1016/0022-2836(80)90291-0

[61] Norton, I.T., Goodall, D.M., Morris, E.R. and Rees, D.A. (1983) Dynamics of Cation-Induced Conformational Ordering in Solution of Segmented Iota-Carrageenan. Journal of Chemical Society, Faraday Transaction, 79, 2501-2515. http://dx.doi.org/10.1039/f19837902501

[62] Ridout, M.J., Garza, S., Brownsey, G.J. and Morris, V.J. (1996) Mixed Iota-Kappa Carrageenan Gels. Internal Journal of Biological Macromolecules, 18, 5-8. http://dx.doi.org/10.1016/0141-8130(95)01037-8

[63] Janaswamy, S. and Chandrasekaran, R. (2003) Effect of Calcium Ions on the Organization of Iota-Carrageenan Helices and X-Ray Investigation. Carbohydrate Research, 337, 523-535. http://dx.doi.org/10.1016/S0008-6215(02)00017-4

[64] Thrimawithana, T.R., Young, S., Dunston, D.E. and Alany, R.G. (2010) Textures and Rheological Characterization of Kappa- and Iota-Carrageenan in the Presence of Counter Ions. Carbohydrate Polymers, 82, 69-77. http://dx.doi.org/10.1016/j.Carbpol.2010.04.024

[65] Patel, B.K., Campanella, O.H. and Janaswamy, S. (2013) Impact of Urea on the Three-Dimensional Structure, Viscosity and Thermal Behavior of Iota-Carrageenan. Carbohydrate Polymers, 92, 1873-1879. http://dx.doi.org/10.1016/j.carbpol.2012.11.026

[66] Arnott, S., Fulmer, A., Scott, W.E., Dea, I.C.M., Moorhouse, R. and Rees, D.A. (1974) The Agarose Double Helix and Its Function in Agarose Gel Structure. Journal of Molecular Biology, 90, 269-284. http://dx.doi.org/10.1016/0022-2836(74)90372-6

[67] Mohammed, Z.H., Hember, M.W.N., Richardson, R.K. and Morris, E.R. (1998) Kinetic and Equilibrium Processes in the Formation and Melting of Agarose Gels. Carbohydrate Polymers, 36, 15-26. http://dx.doi.org/10.1016/S0144-8617(98)00011-3

[68] Cuatrecasas, P., Wilchek, M. and Anfinsen, C.B. (1968) Selective Enzyme Purification by Affinity Chromatography. Proceedings of the National Academy of Sciences of the United States of America, 61, 636-643. http://dx.doi.org/10.1073/pnas.61.2.636

[69] Yon, R.J. (1972) Chromatography of Lipophilic Proteins on Adsorbents Containing Mixed Hydrophobic and Ionic Groups. Biochemical Journal, 126, 765-767.

[70] Gamini, A., Toffanin, R., Murano, E. and Rizzo, R. (1997) Hydrogen Bonding and Conformation of Agarose in Methyl Sulfoxide and Aqueous Solutions Investigated by ${ }^{1} \mathrm{H}$ and ${ }^{13} \mathrm{C}$ NMR Spectroscopy. Carbohydrate Research, 304, 293302. http://dx.doi.org/10.1016/S0008-6215(97)00232-2

[71] Baird, J.K., Sandford, P.A. and Cottrell, I.W. (1983) Industrial Applications of Some New Microbial Polysaccharides. Biotechnology, 1, 778-783. http://dx.doi.org/10.1038/nbt1183-778

[72] Sandford, P.A., Cottrell, I.W. and Pettitt, D.J. (1984) Microbial Polysaccharides: New Products and Their Commercial Applications. Pure and Applied Chemistry, 56, 879-892. http://dx.doi.org/10.1351/pac198456070879

[73] O’Neill, M.A., Selvendran, R.R. and Morris, V.J. (1983) Structure of the Acidic Extracellular Gelling Polysaccharide Produced by Pseudomonas elodea. Carbohydrate Research, 124, 123-133. http://dx.doi.org/10.1016/0008-6215(83)88360-8

[74] Jansson, P.-E., Lindberg, B. and Sandford, P.A. (1983) Structural Studies of Gellan Gum, an Extracellular Polysaccharide Elaborated by Pseudomonas elodea. Carbohydrate Research, 124, 135-139. http://dx.doi.org/10.1016/0008-6215(83)88361-X 
[75] Kuo, M.-S., Mort, A.J. and Dell, A. (1987) Identification and Location of L-Glycerate, an Unusual Acyl Substituent in Gellan Gum. Carbohydrate Research, 156, 173-187. http://dx.doi.org/10.1016/S0008-6215(00)90109-5

[76] Upstill, C., Atkins, E.D.T. and Attwool, P.T. (1986) Helical Conformation of Gellan Gum. International Journal of Biological Macromolecules, 8, 275-288. http://dx.doi.org/10.1016/0141-8130(86)90041-3

[77] Grasdalen, H. and Smidsrod, O. (1987) Gelation of Gellan Gum. Carbohydrate Polymers, 7, 371-393. http://dx.doi.org/10.1016/0144-8617(87)90004-X

[78] Chandrasekaran, R., Pulgjaner, L.C., Joyce, K.L. and Arnott, S. (1988) Cation Interaction in Gellan: An X-Ray Study of the Potassium Salt. Carbohydrate Research, 181, 23-40. http://dx.doi.org/10.1016/0008-6215(88)84020-5

[79] Lee, E.J. and Chandrasekaran, R. (1991) X-Ray and Computer Modeling Studies on Gellan-Related Polymers: Molecular Structures of Welan, S-657, and Rhamsan. Carbohydrate Research, 214, 11-24. http://dx.doi.org/10.1016/S0008-6215(00)90526-3

[80] Morris, E.R., Gothard, M.G.E., Hember, M.W.N., Manning, C.E. and Robinson, G. (1996) Conformation and Rheological Transitions of Welan, Rhamsan and Acylated Gellan. Carbohydrate Polymers, 30, 165-175. http://dx.doi.org/10.1016/S0144-8617(96)00059-8

[81] Jansson, P.-E., Lindberg, B. and Wildmalm, G. (1985) Structural Studies of a Polysaccharide (S-130) Elaborated by Alcaligenes ATCC 31555. Carbohydrate Research, 139, 217-223. http://dx.doi.org/10.1016/0008-6215(85)90022-9

[82] Jansson, P.-E., Lindberg, B., Lindberg, J. and Maekawa, E. (1986) Structural Studies of a Polysaccharide (S-194) Elaborated by Alcaligenes ATCC 31961. Carbohydrate Research, 156, 157-163. http://dx.doi.org/10.1016/S0008-6215(00)90107-1

[83] Moorhouse, R. (1987) Structure Property Relationship of a Family of Microbial Polysaccharides. In: Yalpani, M., Ed., Industrial Polysaccharides, Elsevier Science Publisher, Amsterdam, 187-206.

[84] Chowdhury, T.A., Lindberg, B., Lindouist, U. and Baird, J. (1987) Structure Studies of an Extracellular Polysaccharide, S-657, Elaborated by Xanthomonas ATCC 53159. Carbohydrate Research, 164, 117-122. http://dx.doi.org/10.1016/0008-6215(87)80124-6 
Scientific Research Publishing (SCIRP) is one of the largest Open Access journal publishers. It is currently publishing more than 200 open access, online, peer-reviewed journals covering a wide range of academic disciplines. SCIRP serves the worldwide academic communities and contributes to the progress and application of science with its publication.

Other selected journals from SCIRP are listed as below. Submit your manuscript to us via either submit@scirp.org or Online Submission Portal.
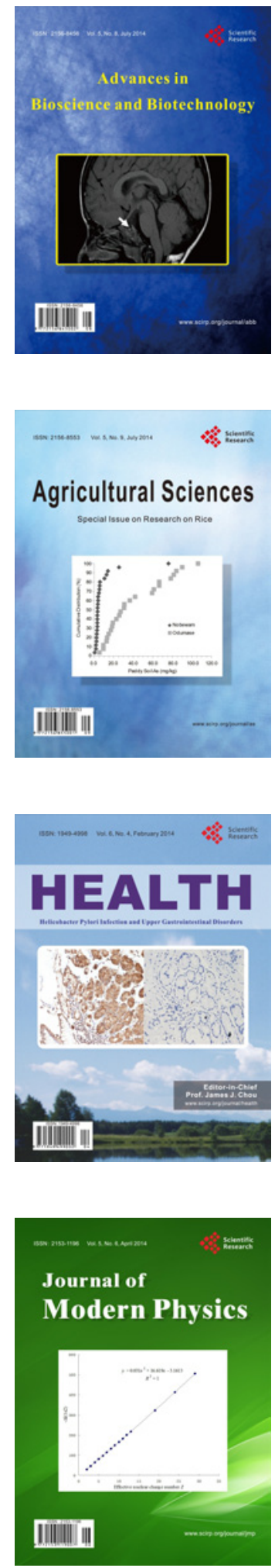
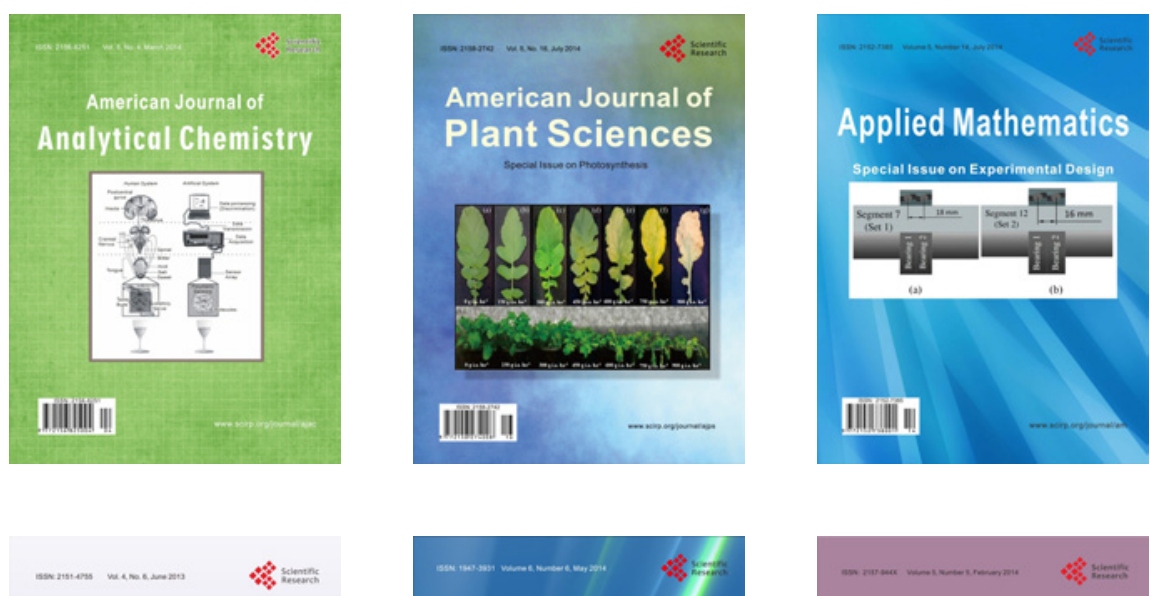

Creative Education
